\title{
Primary renal angiosarcoma: Case report and literature review
}

\author{
Ahmed Chaabouni, MD; Nouri Rebai, MD; Kamel Chabchoub, MD; Mohamed Fourati, MD; Mahdi Bouacida, \\ MD; Mourad Hadj Slimen, MD; Ali Bahloul, MD; Mohamed Nabil Mhiri, MD
}

Department of Urology, Habib Bourguiba University Hospital, Tunisia

Cite as: Can Urol Assoc J 2013;7(5-6):e430-2. http://dx.doi.org/10.5489/cuaj.1396

Published online June 12, 2013.

\section{Abstract}

Angiosarcoma is a rare malignant tumour occurring in less than $2 \%$ of soft tissue sarcomas. Angiosarcoma involving the kidney usually represents metastasis from skin or visceral primary lesions, while angiosarcoma primarily occurring in the kidney is a very rare neoplasm. We report a case of angiosarcoma of the right kidney in a 59-year-old male. The computed tomography scan showed a solid tumour with a low increased density after administration of contrast medium. Histological examination of the piece of nephrectomy confirmed the diagnosis

\section{Introduction}

Angiosarcoma is a rare high-grade malignant tumour accounting for less than $2 \%$ of the soft tissue sarcomas. ${ }^{1}$ The most common site of occurrence of angiosarcomas is the skin (a third of all cases), while the liver, spleen, bone and breast are affected in the remaining two-thirds of patients. Angiosarcoma involving the kidney usually represents metastasis from other primary lesions; primary renal angiosarcoma is extremely rare, with $<40$ cases reported. ${ }^{2}$ We present a case of primary renal angiosarcoma of the kidney in a 59-year-old male.

\section{Case report}

A 59-year-old male presented with a history of right lumbar pain and 3 episodes of macroscopic hematuria, starting 3 months earlier. Physical examination revealed right flank pain. The routine blood tests showed the presence of anemia. An ultrasound showed a large right kidney mass. The remaining blood tests were normal. Abdominal computed tomography confirmed the presence of a tumour in the lower pole of the right kidney measuring $6 \mathrm{~cm}$ in maximum diameter, with a low increased density after administration of contrast medium (Fig. 1, Fig. 2).

A radical nephrectomy, including the ipsilateral adrenal gland, was performed. The macroscopic appearance of the solid tumour measured $6.5 \times 5 \times 4 \mathrm{~cm}$. It was located in the middle of the kidney, and was brown in colour with marked hemorrhage and necrosis. The tumour involved perinephric fatty tissue. The adrenal gland was unremarkable. Histopathological examination showed pleomorphic tumour cells with large hyperchromatic nuclei, prominent nucleoli and clear cytoplasm. An immunohistochemical study showed a vascular tumour strongly and diffusely positive for vimentine, CD31; it was focally positive for factor VIII. The tumour was uniformly negative for CD10, desmin and alpha-smooth muscle actin. These findings indicated that the tumour was angiosarcoma of the kidney. The postoperative course was uneventful and the patient was discharged 4 days after the operation.

On the basis of the histological findings, we decided to administer adjuvant chemotherapy, but this was not done as the patient died a month after surgery.

\section{Discussion}

The present case is an exceptional primary angiosarcoma of the kidney. It is a very rare tumor, and very little information on this entity is available. The present case was characterized by the absence of detectable metastases at diagnosis because most angiosarcomas are already metastasized when the diagnosis was made. ${ }^{3}$ However, despite the only primary localization, our patient's prognosis was similar to the prognosis of angiosarcoma metastasized at diagnosis (2-24 months), confirming that micrometastases had already occurred at diagnosis. ${ }^{3}$

Renal angiosarcoma shows no clinical presentation and patients often present with symptoms for renal disease (i.e., side pain, macroscopic hematuria, palpable abdominal 


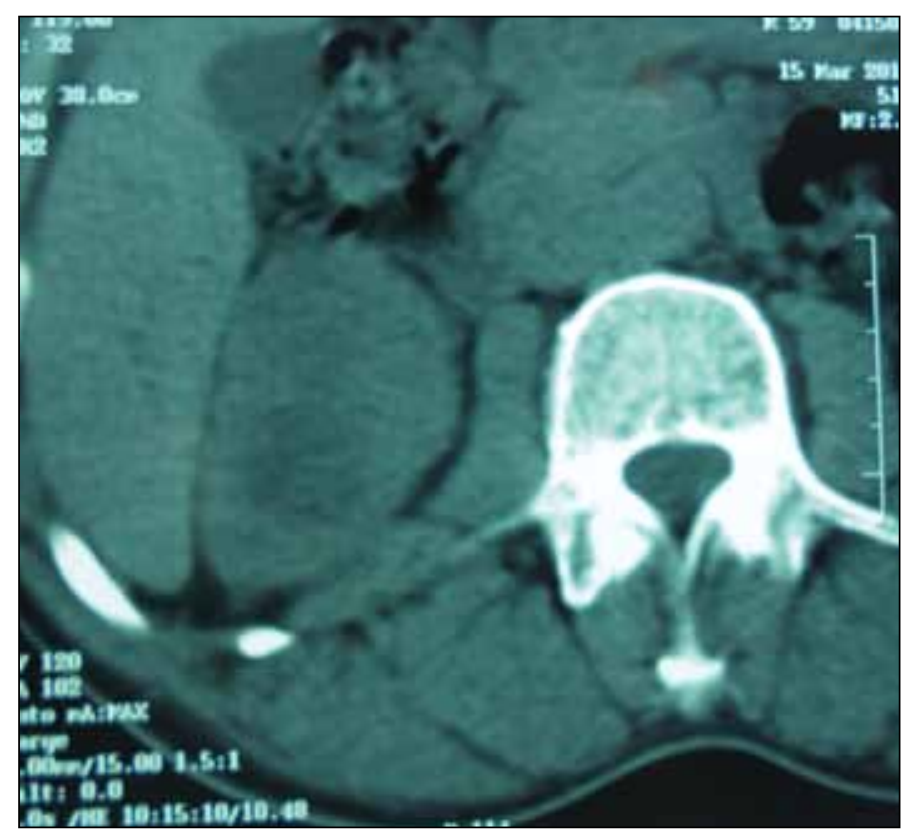

Fig. 1. Computed tomography scan showing solid mass in the lower pole of the right kidney.

mass).$^{4}$ Generally, a computed tomography scan reveals a solid renal tumour with no characteristic signs and studied local and metastases extension. ${ }^{5}$

The prognosis of renal angiosarcoma is uniformly fatal with widespread metastases. Metastases are found mainly in the lung, liver and bones. The most important factor determining the prognosis of renal angiosarcoma in many studies seemed to be the size of the initial lesion. ${ }^{6-8}$ Tumours $<5 \mathrm{~cm}$ in diameter have a significantly better prognosis than larger lesions. Analyzing various cases of angiosarcoma, Mark and colleagues reported a 5 -year survival of $32 \%$ for lesions $<5 \mathrm{~cm}$ compared to $13 \%$ for those $>5 \mathrm{~cm}^{9}{ }^{9}$ Angiosarcoma of the kidney is highly malignant, and there seems to be no standard therapy.

Regarding the possible treatment of kidney angiosarcoma, surgery remains the most effective approach. Radical nephrectomy must be performed in all cases because of the impossibility of making a differential diagnosis between primary renal angiosarcoma and the more common renal cell cancer. ${ }^{2}$ On the other hand, data regarding radiotherapy and/or chemotherapy are controversial. Certainly, the lack of a standard therapy is a result of the rarity of this malignancy. ${ }^{8-10}$ According to Zenico and colleagues, patients who had the best response also underwent radiotherapy and chemotherapy, with a median survival of 13 months $(p>0.005)$ compared to 7 months in patients who only underwent nephrectomy. ${ }^{2}$ However, taxanes may be more efficient than standard chemotherapy in the treatment of metastatic renal angiosarcoma. ${ }^{1}$ Therapy must be specific to each patient, with special attention on the presence of distant metastases. In our case, nephrectomy was

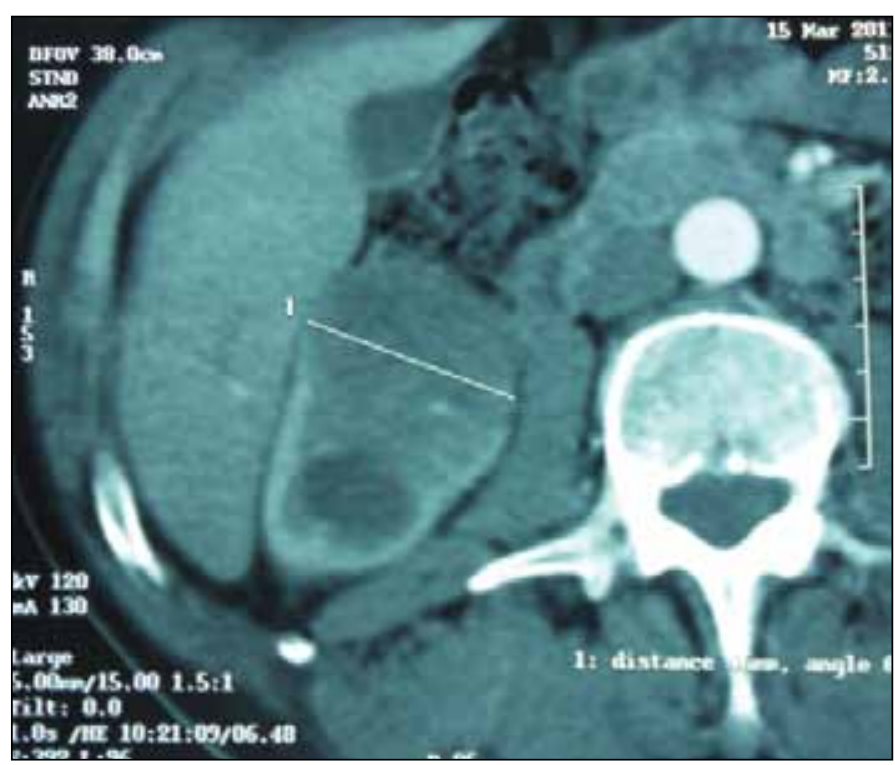

Fig. 2. Abdominal computed tomography scan showing inhomogeneously mass with a low increased density, after administration of contrast medium.

chosen followed by chemotherapy to delay the development of distant metastases, but the patient died before treatment.

\section{Conclusion}

Renal angiosarcoma represents a rare variant of renal parenchymal cancer. It has a high potential of malignancy, with a very short survival period. The very low incidence of this tumour and the extreme variability in its clinical course means that we are still far from defining a diagnostic and therapeutic protocol.

Competing interests: None declared.

This paper has been peer-reviewed.

\section{References}

1. Papadimitriou VD, Stamatiou KN, Takos DM, et al. Angiosarcoma of kidney: A case report and review of literature. Urol J 2009;6:223-5.

2. Zenico T, Saccomanni $M$, Salomone $U$, et al. Primary renal angiosarcoma: case report and review of world literature. Tumori 2011;97:e6-9.

3. Lee $\mathrm{CH}$, Park KU, Nah DY, et al. Bilateral spontaneous pneumothorax during cytotoxic chemotherapy for angiosarcoma of the scalp. J Korean Med Sci 2003;18:277-80.

4. Fukunaga M. Angiosarcoma of the kidney with minute clear cell carcinomas: a case report. Pathol Res Pract 2009;205:347-51. http://dx.doi.org/10.1016/i.prp.2008.11.005

5. Berretta $M$, Rupolo $M$, Buonadonna $A$, et al. Metastatic angiosarcoma of the kidney: a case report with treatment approach and review of the literature. J Chemother 2006;18:221-4.

6. Leggio L. Primary angiosarcoma of the kidney: size is the main prognostic factor. Int J Urol 2007; 14:777-8. http://dx.doi.org/10.1111/j.1442-2042.2007.01785.x

7. Leggio L, Addolorato G, Abenavoli L, et al. Primary renal angiosarcoma: a rare malignancy. A case report and review of the literature. Urol Oncol 2006;24:307-12. http://dx.doi.org/10.1016/j.urolonc.2005.10.002 
Chaabouni et al.

8. Nguyen T, Auquier MA, Renard C, et al. Hemoptysis and spontaneous rupture of a primary renal angiosarcoma: a case report. J Radiol 2010;91:1313-7. http://dx.doi.org/10.1016/S0221-0363(10)70201-8

9. Mark RJ, Poen IC, Tran LM, et al. Angiosarcoma. A report of 67 patients and a review of the literature. Cancer 1996; 77:2400-6. httrp://dx.doi.org/10.1002/(SIC) 1097-0142(19960601)77:11<2400:AID-CNCR32>3.0.C0;2-Z

10. Douard $A$, Pasticier $G$, Deminière $C$, et al. Primary angiosarcoma of the kidney: case report and the literature review. Prog Urol 2012;22:438-41. http://dx.doi.org/10.1016/i.purol.2011.11.006
Correspondence: Dr. Ahmed Chaabouni, Department of Urology, Habib Bourguiba University Hospital, Maijda Boulila Road SFAX 3029, Tunisia; chaabouni.ahmad@gmail.com 